\title{
Factors associated with contraceptive use among young women in Malawi: analysis of the 2015-16 Malawi demographic and health survey data
}

Chrispin Mandiwa ${ }^{1,5^{*}}$, Bernadetta Namondwe ${ }^{2}$, Andrew Makwinja ${ }^{3}$ and Collins Zamawe ${ }^{4}$

\begin{abstract}
Background: Although Malawi is one of the countries with highest Contraceptive Prevalence Rate (CPR) in Sub-Saharan Africa, pregnancies and fertility among young women remain high. This suggests low up take of contraceptives by young women. The aim of this study was to investigate the factors associated with contraceptive use among young women in Malawi.

Methods: This is a secondary analysis of household data for 10,422 young women aged 15-24 years collected during the 2015-16 Malawi Demographic and Health Survey (MDHS). The sample was weighted to ensure representativeness. Descriptive statistics, bivariate and multivariate logistic regressions were performed to assess the demographic, social economic and other factors that influence contraceptive use among young women. Crude Odds Ratio (COR) and Adjusted Odds Ratio (AOR) with their corresponding 95\% confidence intervals (95\% Cl) were computed using the Statistical Package for the Social Sciences version 22.0.

Results: Of the 10,422 young women, 3219 used contraception representing a prevalence of 30.9\%. The findings indicate that age, region of residence, marital status, education, religion, work status, a visit to health facility, and knowledge of the ovulatory cycle are significant predictors of contraceptive use among young women in Malawi. Women who were in the age group 20-24 years ( $A O R=1.93 ; 95 \% \mathrm{Cl}=1.73-2.16$ ), working ( $\mathrm{AOR}=1.26 ; 95 \% \mathrm{Cl}=1.14-1.39$ ), currently married (AOR $=6.26 ; 95 \% \mathrm{Cl}=5.46-7.18)$, knowledgeable about their ovulatory cycle ( $\mathrm{AOR}=1.75 ; 95 \% \mathrm{Cl}=1.50-2.05)$, and those with primary education ( $\mathrm{AOR}=1.47 ; 95 \% \mathrm{Cl}=1.18-1.83$ ) were more likely to use contraceptives than their counterparts.

Conclusion: This study has demonstrated that several social demographic and economic factors are associated with contraceptive use among young women in Malawi. These findings should be considered and reflected in public health policies to address issues that could be barriers to the use of contraception by young women. Strengthening access to family planning information and services for young women is highly recommended to reduce pregnancies among young women in Malawi.
\end{abstract}

Keywords: Contraceptive use, Young women, Family planning, Malawi

\footnotetext{
* Correspondence: crismandiwa@yahoo.com

${ }^{1}$ Ministry of Health, South-West Zone Health Support Office, P.O. Box 3,

Blantyre, Malawi

${ }^{5}$ Malawi Health Sector Programme (DFID Project), Lilongwe, Malawi

Full list of author information is available at the end of the article
}

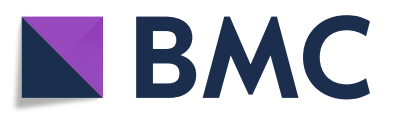

(c) The Author(s). 2018 Open Access This article is distributed under the terms of the Creative Commons Attribution 4.0 International License (http://creativecommons.org/licenses/by/4.0/), which permits unrestricted use, distribution, and reproduction in any medium, provided you give appropriate credit to the original author(s) and the source, provide a link to the Creative Commons license, and indicate if changes were made. The Creative Commons Public Domain Dedication waiver (http://creativecommons.org/publicdomain/zero/1.0/) applies to the data made available in this article, unless otherwise stated. 


\section{Background}

Malawi is among the countries classified by the World Health Organisation (WHO) to have made no progress towards reducing maternal mortality ratio (MMR) between 1990 and 2015 [1]. The country's MMR is currently estimated to be 439 per 100,000 live births, which is one of the highest in Sub-Saharan Africa (SSS) [2]. Many studies have shown that unintended pregnancies among young women greatly contribute to high maternal and neonatal mortality through increased risk for unsafe abortion, birth injuries and postpartum depression [3-6]. Thus, delaying or avoiding pregnancies among young women is a key intervention in preventing and reducing maternal deaths more especially in countries with high maternal mortality like Malawi.

Family planning (the use of modern contraceptives or traditional methods to limit or space pregnancies) is one of the globally recognized essential strategies for reducing maternal and neonatal mortality, particularly in developing countries where almost all maternal and child mortality occur [7-9]. Family planning reduces mortality risk by preventing (a) unintended pregnancies (thereby reducing maternal deaths caused by unsafe abortion), (b) pregnancy among adolescents (who are at a higher risk of death from childbearing), and (c) closely spaced pregnancies (which improves perinatal outcomes and child survival) [10-12]. Therefore, the role of family planning in reducing maternal morbidity and mortality cannot be overemphasized.

Although Malawi is one of the countries with highest Contraceptive Prevalence Rate (CPR) in Sub-Saharan Africa, pregnancies among young women and fertility remain high [2]. A recent Malawi Demographic Health Survey (MDHS) report indicates that teenage childbearing has increased by 3\% between 2010 and 2016, which suggests low use of contraceptives by young women [2]. The determinants of contraceptive use have been explored around the world among women of child bearing age (15-49 years old), but data on the use by young women is limited [13-15]. Understanding the key factors influencing contraceptive use among young women who are at a higher risk of maternal mortality and morbidity could inform interventions to improve uptake of contraceptives among this group. Therefore, the aim of this study was to examine the correlates of contraceptive use among young women in Malawi.

\section{Methods}

\section{Study design and data source}

This is a secondary analysis of cross-sectional household data for women collected during the 2015-16 MDHS. Four questionnaires were used for the data collection: the Household Questionnaire, the Women's Questionnaire, the Men's Questionnaire and the Biomarker
Questionnaire. The data used in this analysis were collected using the women's questionnaire.

\section{Overview of the MDHS: objectives, population and sampling}

The MDHS is a nationwide survey with a representative sample of women and men aged 15-49 and 15-54, respectively. It is designed to provide data for monitoring the population and health situation in Malawi [2].

A two-stage cluster sampling procedure was used to generate a nationally representative sample of households. In the first stage, 850 enumeration areas or clusters (173 clusters in urban areas and 677 in rural areas) were selected with probability proportional to sample enumeration area (SEA) size. In the second stage, 30 households per urban cluster and 33 per rural cluster were selected using a systematic random sampling approach. All women of reproductive age (15-49 years) in the selected households were eligible to participate. In the 850 selected clusters, 26,564 households were occupied at the time of data collection of which 26,361 were successfully interviewed, yielding a household response rate of $99 \%$. In total, 24,562 women were successfully interviewed and in this analysis we have included 10,422 young women aged 15-24 years.

\section{Study variables and measurements Dependent variable}

The outcome variable of this study was contraceptive use. Data on contraceptive use was obtained through the women's questionnaire. Women were asked this question: "Are you or your partner currently doing something or using any method to delay or avoid getting pregnant?" Women who reported current use of either modern or traditional contraceptive methods were considered as current users of contraceptives and those who responded with a 'no' were regarded as non-users.

\section{Independent variables}

The variables were grouped into three categories; sociodemographic variables which included age (in two categories; 15-19 and 20-24), marital status (in three categories; never married, currently married, formerly married), religion (in four categories; catholic, other Christian, Muslim, no religion), region (in three categories; northern, central, south), residence (urban/rural); social-economic variables which included education (in three categories; none, primary school, secondary school or above), wealth index (in three categories; poor, medium, rich) and work status/paid work (working/not working). The other independent factors were participant's knowledge of ovulatory cycle (yes/no) and woman's visit to a health facility. 


\section{Ethical consideration}

Permission to use the data was obtained from the MEASURE DHS, which is the monitoring and evaluation body of the demographic health survey (DHS) globally. The original study obtained ethical clearance from the Malawi's National Health Sciences Research Committee (NHSRC). All participants provided oral informed consent.

\section{Data management and analysis}

First, we cleaned the data and recoded some of the variables to suit the objective of this study. Descriptive statistics was used to summarize the data and the results were presented as proportions (\%). Bivariate analyses (Pearson $X^{2}$ square) were conducted to determine the associations between contraceptive use and each of the predictor variables. Variables that had an association with contraceptive use at $\leq 0.25$ on binary logistic regression were further analysed using multivariate logistic regression to identify predictors of contraceptive use [16]. Crude and adjusted odds ratios and their 95\% confidence intervals $(95 \% \mathrm{CI})$ were estimated. To adjust for clustering, all statistical analyses were performed using complex samples analysis of the Statistical Package for the Social Sciences (SPSS, IBM version 22), and statistical significance was set at $P$-value of less than 0.05 . All analyses were weighted using a sample weight that was generated for the dataset to account for differences in sampling probabilities.

\section{Results}

\section{Social demographic characteristics of the study}

\section{participants}

Of the 10,422 (weighted) eligible participants, 3219 used contraception representing a prevalence of $30.9 \%$. Table 1 summarises the characteristics of the study participants. In brief, about half (50.5\%) were in the 15-19 years age group while $49.5 \%$ were aged 20 to 24 and the majority $(81.8 \%)$ of the participants were rural dwellers. Nearly half $(45.4 \%)$ of the participants were from southern region and $46.9 \%$ were married. Overall, $18.5 \%$ of the participants were Catholics while $12.9 \%$ were Muslims and only $0.2 \%$ had no religion. Over $60 \%$ of the participants had attained primary school education, $49.4 \%$ were working and $40.3 \%$ were from poor households. Majority $(82.7 \%)$ of the participants had knowledge of their ovulatory cycle and over half $(58.1 \%)$ of the participants did not visit any health facility.

\section{Factors associated with contraceptive use}

Overall, most of the social and demographic characteristics of participants were significantly associated with contraceptive use (Tables 1 and 2). We found that women in the age group 20-24 years were 93\% (AOR =
1.93; $95 \% \mathrm{CI}=1.73-2.16)$ more likely to use contraceptives compared to adolescents in the age group 1519 years. Married $(\mathrm{AOR}=6.26 ; 95 \% \mathrm{CI}=5.46-7.18)$ and formerly married (AOR $=3.94 ; 95 \% \mathrm{CI}=3.23-4.81)$ participants had higher odds to use contraceptives than their counterparts who were unmarried. Women who were from central $(\mathrm{AOR}=1.22 ; 95 \% \mathrm{CI}=1.03-1.43])$ and southern region $(\mathrm{AOR}=1.29 ; 95 \% \mathrm{CI}=1.09-1.51)$ were more likely to use contraceptives than those who were from northern region. In addition, women who had knowledge of their ovulatory cycle had $75 \%$ higher odds $(\mathrm{AOR}=1.75 ; 95 \% \mathrm{CI}=1.50-2.05)$ to use contraceptives than their counterparts who had no knowledge of their ovulatory cycle. Likewise, women who visited a health facility were $61 \%(\mathrm{AOR}=1.61 ; 95 \% \mathrm{CI}=1.45-1.79)$ more likely to use contraceptives than their counterparts who had not visited any health facility. Women who attained primary school education were $47 \%(\mathrm{AOR}=1.47 ; 95 \%$ $\mathrm{CI}=1.18-1.83)$ more likely to use contraceptives than uneducated women. On the other hand, Muslim young women had $49 \%$ ( $\mathrm{AOR}=0.51 ; 95 \% \mathrm{CI}=0.43-0.61)$ lesser odds of using contraceptive than Catholic women. Moreover, women who were rural dwellers were $24 \%$ (AOR $=0.76 ; 95 \% \mathrm{CI}=0.65-0.88)$ less likely to use contraceptives than those who were urban dwellers.

The distribution of sources of information for contraceptive methods for young women was assessed graphically. Majority of the participants $(90.3 \%$ ) heard about contraceptive methods from health field workers while only $4.2 \%$ got information for contraceptive methods from mobile text messages as shown in Fig. 1.

\section{Discussion}

The findings indicate that most of the social, economic and demographic characteristics are significant predictors of contraceptive use among adolescents and young women in Malawi. We observed that women in the age bracket of 20-24 years were more likely to use contraceptives than their counterparts aged 15-19 years. This observation could partly explain the rise in Malawi's teenage childbearing from $26 \%$ in 2010 to $29 \%$ in 2016 [2] . It is assumed that women aged 20-24 years understand the consequences of engaging in unprotected sexual act or without contraceptive use compared to adolescents. Additionally, most of women aged 1519 years might be newly married, and they may take marriage as an institution of producing children. Adolescents may also have problems in accessing FP services because they may not know where to obtain contraception or cannot afford services. Our results concurs with the findings of previous studies in Ethiopia, Nepal and Uganda which also reported reduced contraceptive use among adolescents compared to women aged 20-24 years [17-19] . 
Table 1 Bivariate association between contraceptive use and various background characteristics

\begin{tabular}{|c|c|c|c|c|c|c|c|}
\hline \multirow[t]{3}{*}{ Characteristics } & & & \multicolumn{4}{|c|}{ Contraceptive utilization } & \multirow{3}{*}{$p$-value } \\
\hline & \multicolumn{2}{|l|}{$\overline{\text { Total }}$} & \multicolumn{2}{|c|}{ Non-users } & \multicolumn{2}{|l|}{ Users } & \\
\hline & $\mathrm{n}$ & $\%$ & $\mathrm{n}$ & $\%$ & $n$ & $\%$ & \\
\hline Age (years) & & & & & & & $<0.001^{*}$ \\
\hline $15-19$ & 5263 & 50.5 & 4452 & 61.8 & 811 & 25.2 & \\
\hline $20-24$ & 5159 & 49.5 & 2751 & 38.2 & 2408 & 74.8 & \\
\hline Residence & & & & & & & $<0.001^{*}$ \\
\hline Rural & 8530 & 81.8 & 5833 & 81.0 & 2697 & 83.8 & \\
\hline Urban & 1892 & 18.2 & 1370 & 19.0 & 522 & 16.2 & \\
\hline Region & & & & & & & $0.236^{* *}$ \\
\hline Northern & 1159 & 11.1 & 818 & 11.4 & 341 & 10.6 & \\
\hline Central & 4536 & 43.5 & 3099 & 43.0 & 1437 & 44.7 & \\
\hline Southern & 4726 & 45.4 & 3286 & 45.6 & 1440 & 44.7 & \\
\hline Marital Status & & & & & & & $<0.001^{*}$ \\
\hline Never married & 4828 & 46.3 & 4394 & 61.0 & 434 & 13.5 & \\
\hline Currently married & 4888 & 46.9 & 2387 & 33.1 & 2501 & 77.7 & \\
\hline Formerly married & 707 & 6.8 & 423 & 5.9 & 284 & 8.8 & \\
\hline Religion & & & & & & & $<0.001^{*}$ \\
\hline Catholic & 1925 & 18.5 & 1336 & 18.5 & 589 & 18.3 & \\
\hline Other Christians & 7131 & 68.4 & 4855 & 67.4 & 2276 & 70.7 & \\
\hline Muslim & 1341 & 12.9 & 1000 & 13.9 & 341 & 10.6 & \\
\hline No religion & 24 & 0.2 & 12 & 0.2 & 12 & 0.4 & \\
\hline Wealth & & & & & & & $<0.001^{*}$ \\
\hline Poor & 4200 & 40.3 & 2660 & 36.9 & 1540 & 47.9 & \\
\hline Medium & 1944 & 18.7 & 1339 & 18.6 & 605 & 18.8 & \\
\hline Rich & 4276 & 41.0 & 3203 & 44.5 & 1073 & 33.3 & \\
\hline Education & & & & & & & $<0.001^{*}$ \\
\hline None & 455 & 4.4 & 298 & 4.1 & 157 & 4.9 & \\
\hline Primary school & 6739 & 64.7 & 4507 & 62.6 & 2232 & 69.4 & \\
\hline Secondary school or above & 3227 & 31.0 & 2398 & 33.3 & 829 & 25.8 & \\
\hline Work status & & & & & & & $<0.001^{*}$ \\
\hline Working & 5145 & 49.4 & 3176 & 44.1 & 1969 & 61.2 & \\
\hline Not working & 5277 & 50.6 & 4027 & 55.9 & 1250 & 38.8 & \\
\hline Rich & & & & & & & \\
\hline Visited health facility & & & & & & & $<0.001^{*}$ \\
\hline Yes & 5650 & 54.2 & 3328 & 46.2 & 2322 & 72.1 & \\
\hline No & 4772 & 45.8 & 3875 & 53.8 & 897 & 27.9 & \\
\hline Knowledge of ovulatory cycle & & & & & & & $<0.001^{*}$ \\
\hline Yes & 8619 & 82.7 & 5654 & 78.5 & 2965 & 92.1 & \\
\hline No & 1803 & 17.3 & 1549 & 21.5 & 254 & 7.9 & \\
\hline
\end{tabular}

${ }^{\bar{*}}$ Significant $(P<0.05) ;{ }^{* *}$ Non-significant $(P>0.05)$

The results further showed disparities in contraceptive use among women by region (southern, central and northern) and residence (Urban vs. Rural). For example, women in central and southern region were more likely to use contraceptives than those in northern region. Similarly, women in rural areas were less likely to use contraceptives than women in urban areas. This is in agreement with results from studies conducted in other 
Table 2 Logistic regression of correlates of contraceptive use by young women in Malawi

\begin{tabular}{|c|c|c|c|c|c|c|}
\hline Characteristics & COR & $95 \% \mathrm{Cl}$ & $P$-value & AOR & $95 \% \mathrm{Cl}$ & $P$-value \\
\hline \multicolumn{7}{|l|}{ Age (years) } \\
\hline $15-19$ & Ref. & & & Ref. & & \\
\hline $20-24$ & 4.81 & $4.38-5.27$ & $<0.001^{*}$ & 1.93 & $1.73-2.16$ & $<0.001^{*}$ \\
\hline \multicolumn{7}{|l|}{ Residence } \\
\hline Urban & Ref. & & & Ref. & & \\
\hline Rural & 1.21 & $1.09-1.36$ & $<0.001^{*}$ & 0.76 & $0.65-0.88$ & $<0.001^{*}$ \\
\hline \multicolumn{7}{|l|}{ Region } \\
\hline Northern & Ref. & & & Ref. & & \\
\hline Central & 1.11 & $0.97-1.28$ & $0.141^{* *}$ & 1.22 & $1.03-1.43$ & $0.020^{*}$ \\
\hline Southern & 1.05 & $0.91-1.21$ & $0.496^{* *}$ & 1.29 & $1.09-1.51$ & $0.003^{*}$ \\
\hline \multicolumn{7}{|l|}{ Marital Status } \\
\hline Never married & Ref. & & & Ref. & & \\
\hline Currently married & 10.62 & $9.48-11.89$ & $<0.001^{*}$ & 6.26 & $5.46-7.18$ & $<0.001^{*}$ \\
\hline Formerly married & 6.81 & $5.69-8.15$ & $<0.001^{*}$ & 3.94 & $3.23-4.81$ & $<0.001^{*}$ \\
\hline \multicolumn{7}{|l|}{ Religion } \\
\hline Catholic & Ref. & & & Ref. & & \\
\hline Other Christians & 1.06 & $0.95-1.19$ & $0.27^{* *}$ & 0.87 & $0.77-0.99$ & $0.034^{*}$ \\
\hline Muslim & 0.77 & $0.66-0.91$ & $<0.001^{*}$ & 0.51 & $0.43-0.61$ & $<0.001^{*}$ \\
\hline No religion & 2.40 & $1.07-5.37$ & $0.033^{*}$ & 1.66 & $0.64-4.30$ & $0.278^{* *}$ \\
\hline \multicolumn{7}{|l|}{ Wealth } \\
\hline Poor & Ref. & & & Ref. & & \\
\hline Medium & 0.78 & $0.70-0.88$ & $<0.001^{*}$ & 0.99 & $0.87-1.13$ & $0.930^{* *}$ \\
\hline Rich & 0.58 & $0.53-0.64$ & $<0.001^{*}$ & 0.93 & $0.82-1.06$ & $0.278^{* *}$ \\
\hline \multicolumn{7}{|l|}{ Education } \\
\hline None & Ref. & & & Ref. & & \\
\hline Primary school & 0.94 & $0.77-1.15$ & $0.542^{* *}$ & 1.47 & $1.18-1.83$ & $<0.001^{*}$ \\
\hline Secondary school or above & 0.66 & $0.53-0.81$ & $<0.001^{*}$ & 1.24 & $0.97-1.57$ & $0.083^{* *}$ \\
\hline \multicolumn{7}{|l|}{ Work status } \\
\hline Not working & Ref. & & & Ref. & & \\
\hline Working & 2.00 & $1.84-2.17$ & $<0.001^{*}$ & 1.26 & $1.14-1.39$ & $<0.001^{*}$ \\
\hline \multicolumn{7}{|l|}{ Visited health facility } \\
\hline No & Ref. & & & Ref. & & \\
\hline Yes & 3.02 & $2.76-3.30$ & $<0.001^{*}$ & 1.61 & $1.45-1.79$ & $<0.001^{*}$ \\
\hline \multicolumn{7}{|l|}{ Knowledge of ovulatory cycle } \\
\hline No & Ref. & & & Ref. & & \\
\hline Yes & 3.20 & $2.78-3.68$ & $<0.001^{*}$ & 1.75 & $1.50-2.05$ & $<0.001^{*}$ \\
\hline
\end{tabular}

$A O R$ adjusted odds ratio, $C O R$ crude odds ratio, Ref reference category

"Significant $(P<0.05) ;{ }^{* *}$ Non-significant $(P>0.05)$

countries, which reported that women living in urban area were more likely to use contraceptives and experience delayed age at marriage than women living in rural area [20-22]. Differences in cultural beliefs and values between rural and urban as well as across regions are some of the possible explanations for the observed disparities in contraceptive use. Moreover, limited availability of health facilities in rural area might further limit access to and use of contraceptives [23]. Thus, program implementers should consider these issues when designing contraceptive programmes for young women. Besides, this highlights the need for further qualitative studies to investigate the actual reasons for these observed variations. 


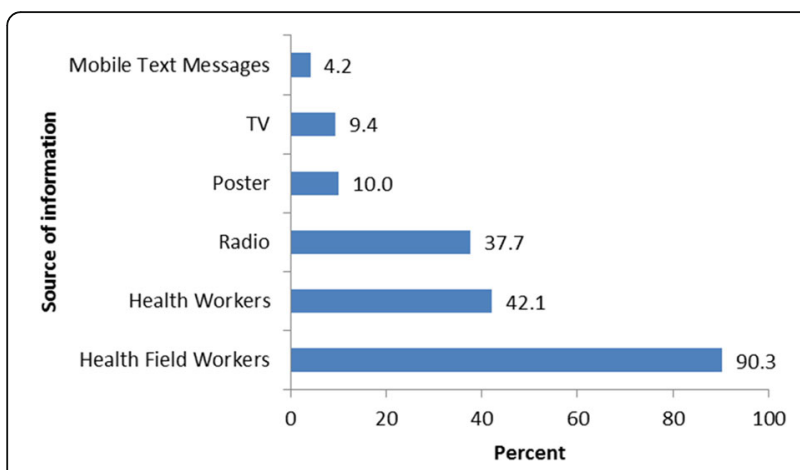

Fig. 1 Distribution of sources of contraceptive methods information for young women in Malawi

The findings also show that women who had attained primary school education had higher odds of using contraceptives than their uneducated counterparts. This finding is consistent with previous studies that have shown a similar pattern of relationship between educational status and contraceptive use [24-28]. Education empowers women to have autonomy in making important decisions regarding fertility related issues and also help them exercise reproductive health rights compared to uneducated women. Moreover, educated women could probably have a better understanding of the benefits of using contraception to reduce unintended pregnancies than women with no education. For that reason, it is necessary that family planning service providers must place special emphasis on, and address the needs of women with no or little education during family planning sessions to provide them with basic reproductive health knowledge to improve uptake of contraceptives. Moreover, it is important for policy makers in Malawi to formulate and enforce policies that promote education of girls and women.

We further observed that work status was a significant predictor of contraceptive use among participants as women who were working were more likely to use contraceptives than women who were not working. The possible explanation for this relationship is that women who are working are too preoccupied with work related activities to having babies as a result they may use contraceptives. Additionally, women who are working are likely to be educated, exposed to contraceptive information and may be able to afford contraceptives than those who are not working. Therefore, there is a need for collaborated efforts by government and its partners to make contraceptives affordable to all women. Our finding is consistent with studies done in other countries which also reported that women who were working were more likely to use contraceptive [29-31].

It was also noted that knowledge of ovulatory cycle had a positive significant relationship with use of contraceptives by young women in Malawi. It is possible that women who know their ovulatory cycle may use contraception methods to protect themselves from getting pregnancy during their ovulation period than their counterparts who do not know their ovulatory cycle. This finding is in agreement with results from a study in Ghana, which reported that women who knew their ovulatory cycle were likely to use contraceptives compared to those who did not know their cycle [29].

We observed a positive significant association between a visit to a health facility and contraceptive use. Perhaps, women who visit health facilities have access or are exposed to sexual and reproductive health services than those who do not visit such facilities. Besides, women who want to or are using contraceptives may also likely visit a health facility. This finding concurs with a recent study in Ethiopia which has reported that women who visited a health facility had 54\% higher odds of using contraceptives [32]. The results of the present study also indicate that majority of young women heard about contraceptive methods from health field workers. This finding is consistent with a study in Bangladesh which reported that many young women mentioned health field workers as a primary source of information for contraceptive methods. Provision of adequate and correct information on contraception to women can positively impact the utilisation of contraceptives and may reduce unintended pregnancy [33]. Thus, a focus on field health workers through outreach visits can improve uptake of contraceptives among young women.

Even though other studies have suggested that contraceptive use is associated with wealth index, [34-36] our study did not. These conflicting results could possibly be due to different sample size, study participants and setting.

The findings of this study should be considered in light of the following limitations and strengths. First, we used secondary data and some important independent variables of contraceptive use were not available. Second, DHS is cross-sectional in nature as such we cannot establish temporal linkages. Third, contraceptive use was based on self-reported. So, recall bias cannot be ruled out. Fourth, this study did not differentiate the types of contraception (modern and traditional) during analysis. Independent variables may have different influence on the type of contraception. Nevertheless, the main interest in our study was identifying the determinants of contraceptive use among young women in general, not on a specific type of contraception. Future studies need to be conducted to assess if the variables have different or similar effects on modern versus traditional contraceptive methods. The strengths of this study include the use of nationally representative data with relatively large sample size, which imply robust statistical significance. 


\section{Conclusion}

The findings of this study highlight the influence of age, type of residence, region of residence, marital status, education, religion, a visit to health facility, work status and knowledge of the ovulatory cycle as key predictors of contraceptive use among young women in Malawi. These findings should be considered and reflected in public health policies to address issues that could be barriers to the use of contraception by young women. Improving access and use of contraception is highly recommended to reduce teenage pregnancies in Malawi.

\section{Abbreviations}

CPR: Contraceptive prevalence rate; MDHS: Malawi demographic health survey; MMR: Maternal mortality ratio; NSO: National statistical office; SSS: Sub-Saharan Africa; STI: Sexually transmitted infections; WHO: World Health Organisation

\section{Acknowledgements}

Many thanks to the DHS programme team for allowing us to use the dataset.

\section{Availability of data and materials}

The datasets used and/or analysed during the current study are available upon request from the Demographic Health Survey Programme Team. In addition, the datasets can be obtained from the corresponding author on reasonable request.

\section{Authors' contributions}

CM and CZ conceived and designed the study. CM developed the study protocol and requested data from the DHS programme. CM performed the data analysis, interpretation of data and drafted the manuscript. AM, BN and CZ critically revised the draft manuscript. All authors read and approved the final manuscript.

\section{Authors' information}

$\mathrm{CM}$ has MPH and is a Monitoring and Evaluation Advisor at Malawi Ministry of Health, South-West Zone Quality Management Office in Blantyre. AM has BSc in Nursing and he is studying towards MPH at University of Malawi. BN has BSC in Nursing and midwifery from University of Malawi - Kamuzu College of Nursing. CZ is a PhD student at University College London in UK.

\section{Ethics approval and consent to participate}

Permission to use the data was obtained from the MEASURE DHS, which is the monitoring and evaluation body of the demographic health survey (DHS) globally. The original study obtained ethical clearance from the Malawi's National Health Sciences Research Committee (NHSRC). All participants provided oral informed consent.

\section{Competing interests}

The authors declare that they have no competing interests.

\section{Publisher's Note}

Springer Nature remains neutral with regard to jurisdictional claims in published maps and institutional affiliations.

\section{Author details}

${ }^{1}$ Ministry of Health, South-West Zone Health Support Office, P.O. Box 3 , Blantyre, Malawi. ${ }^{2}$ University of Malawi, Kamuzu College of Nursing, Lilongwe, Malawi. ${ }^{3}$ University of Malawi, College of Medicine, Blantyre, Malawi. ${ }^{4}$ University College London, Institute for Global Health, London, UK. ${ }^{5}$ Malawi Health Sector Programme (DFID Project), Lilongwe, Malawi.
Received: 29 January 2018 Accepted: 15 May 2018

Published online: 20 September 2018

\section{References}

1. Simwaka BN, Theobald S, Amekudzi YP, Tolhurst R. Meeting millenium development goals 3 and 5: gender equality needs to be put on the African agenda. Brit Med J. 2005;331(7519):708-9.

2. ICF NSONMa. Malawi demographic and health survey 2015-16. Zomba and Rockville: NSO and ICF; 2017

3. Ganchimeg T, Ota E, Morisaki N, Laopaiboon M, Lumbiganon P, Zhang J, et al. Pregnancy and childbirth outcomes among adolescent mothers: a World Health Organization multicountry study. BJOG. 2014;121(Suppl 1):40-8. https://doi.org/10.1111/1471-0528.12630.

4. Olson RM, Kamurari S. Barriers to safe abortion access: uterine rupture as complication of unsafe abortion in a Ugandan girl. BMJ Case Rep. 2017;2017 https://doi.org/10.1136/bcr-2017-222360.

5. Vallely LM, Homiehombo P, Kelly-Hanku A, Whittaker A. Unsafe abortion requiring hospital admission in the Eastern Highlands of Papua New Guinea-a descriptive study of women's and health care workers' experiences. Reprod Health. 2015:12:22. https://doi.org/10.1186/s12978-015-0015-x.

6. Abbasi S, Chuang CH, Dagher R, Zhu J, Kjerulff K. Unintended pregnancy and postpartum depression among first-time mothers. J Women's Health (2002). 2013;22(5):412-6. https://doi.org/10.1089/jwh.2012.3926.

7. Ahmed S, Li Q, Liu L, Tsui AO. Maternal deaths averted by contraceptive use: an analysis of 172 countries. Lancet. 2012;380(9837):111-25. https://doi. org/10.1016/s0140-6736(12)60478-4

8. Ganatra B, Faundes A. Role of birth spacing, family planning services, safe abortion services and post-abortion care in reducing maternal mortality. Best Pract Res Clin Obstet Gynaecol. 2016;36:145-55. https://doi.org/10. 1016/j.bpobgyn.2016.07.008.

9. Cleland J, Conde-Agudelo A, Peterson H, Ross J, Tsui A: Contraception and health. Lancet. 2012;380(9837):149-56

10. Canning D, Schultz TP. The economic consequences of reproductive health and family planning. Lancet. 2012;380(9837):165-71.

11. Diamond-Smith N, Potts M. A woman cannot die from a pregnancy she does not have. Int Perspect Sex Reprod Health. 2011;37(3):155-7.

12. Stover J, Ross J. How increased contraceptive use has reduced maternal mortality. Matern Child Health J. 2010;14(5):687-95.

13. Palamuleni ME. Socio-economic and demographic factors affecting contraceptive use in Malawi. Afr J Reprod Health. 2013;17(3):91-104.

14. Lakew Y, Reda AA, Tamene H, Benedict S, Deribe K. Geographical variation and factors influencing modern contraceptive use among married women in Ethiopia: evidence from a national population based survey. Reprod Health. 2013:10(1):52.

15. Medhanyie AA, Desta A, Alemayehu M, Gebrehiwot T, Abraha TA, Abrha A, et al. Factors associated with contraceptive use in Tigray, North Ethiopia. Reprod Health. 2017;14(1):27.

16. Sun G-W, Shook TL, Kay GL. Inappropriate use of bivariable analysis to screen risk factors for use in multivariable analysis. J Clin Epidemiol. 1996:49(8):907-16.

17. Binu W, Marama T, Gerbaba M, Sinaga M. Sexual and reproductive health services utilization and associated factors among secondary school students in Nekemte town, Ethiopia. Reprod Health. 2018;15(1):64. https://doi.org/10. 1186/s12978-018-0501-z.

18. Tamang L, Raynes-Greenow C, McGeechan K, Black K. Factors associated with contraceptive use among sexually active Nepalese youths in the Kathmandu Valley. Contracept Reprod Med. 2017;2:13. https://doi.org/10. 1186/s40834-017-0040-y

19. Asiimwe JB, Ndugga P, Mushomi J, Manyenye Ntozi JP. Factors associated with modern contraceptive use among young and older women in Uganda; a comparative analysis. BMC Public Health. 2014;14:926. https://doi. org/10.1186/1471-2458-14-926

20. Alemayehu T, Haider J, Habte D. Determinants of adolescent fertility in Ethiopia. Ethiop J Health Dev. 2010;24(1):e3086.

21. White JS, Speizer IS. Can family planning outreach bridge the urban-rural divide in Zambia? BMC Health Serv Res. 2007:7(1):143.

22. Pradhan $R$, Wynter $K$, Fisher J. Factors associated with pregnancy among adolescents in low-income and lower middle-income countries: a systematic review. J Epidemiol Community Health. 2015;69(9):918-24.

23. Abiiro GA, Mbera GB, De Allegri M. Gaps in universal health coverage in Malawi: a qualitative study in rural communities. BMC Health Serv Res. 2014;14(1):234. 
24. Tekelab T, Melka AS, Wirtu D. Predictors of modern contraceptive methods use among married women of reproductive age groups in western Ethiopia: a community based cross-sectional study. BMC Womens Health. 2015;15:52. https://doi.org/10.1186/s12905-015-0208-z.

25. Mekonnen W, Worku A. Determinants of low family planning use and high unmet need in Butajira District, south Central Ethiopia. Reprod Health. 2011; 8:37. https://doi.org/10.1186/1742-4755-8-37.

26. Babalola S, Fatusi A. Determinants of use of maternal health services in Nigeria-looking beyond individual and household factors. BMC Pregnancy Childbirth. 2009;9:43. https://doi.org/10.1186/1471-2393-9-43.

27. Adanu RM, Seffah JD, Hill AG, Darko R, Duda RB, Anarfi JK. Contraceptive use by women in Accra, Ghana: results from the 2003 Accra Women's Health Survey. Afr J Reprod Health. 2009;13(1):123-33.

28. Nketiah-Amponsah E, Arthur E, Aaron A. Correlates of contraceptive use among Ghanaian women of reproductive age (15-49 years). Afr J Reprod Health. 2012;16(3):155-70.

29. Nyarko SH. Prevalence and correlates of contraceptive use among female adolescents in Ghana. BMC Womens Health. 2015;15(1):60.

30. Jalang'o R, Thuita F, Barasa SO, Njoroge P. Determinants of contraceptive use among postpartum women in a county hospital in rural KENYA. BMC Public Health. 2017;17(1):604. https://doi.org/10.1186/s12889-017-4510-6.

31. Wuni C, Turpin CA, Dassah ET. Determinants of contraceptive use and future contraceptive intentions of women attending child welfare clinics in urban Ghana. BMC Public Health. 2017:18(1):79. https://doi.org/10.1186/ s12889-017-4641-9.

32. Ebrahim NB, Atteraya MS. Structural correlates of modern contraceptive use among Ethiopian women. Health Care Women Int. 2018;39(2):208-19. https://doi.org/10.1080/07399332.2017.1383993.

33. Lee JK, Parisi SM, Akers AY, Borrerro S, Schwarz EB. The impact of contraceptive counseling in primary care on contraceptive use. J Gen Intern Med. 2011;26(7):731-6.

34. Kanwal Aslam S, Zaheer S, Qureshi MS, Aslam SN, Shafique K. Socioeconomic disparities in use of family planning methods among Pakistani women: findings from Pakistan demographic and health surveys. PLoS One. 2016;11(4):e0153313. https://doi.org/10.1371/journal.pone.0153313.

35. Mekonnen FA, Mekonnen WN, Beshah SH. Predictors of long acting and permanent contraceptive methods utilization among women in Rural North Shoa, Ethiopia. Contracept Reprod Med. 2017;2:22. https://doi.org/10.1186/ s40834-017-0049-2.

36. Rasooly MH, Ali MM, Brown NJ, Noormal B. Uptake and predictors of contraceptive use in Afghan women. BMC Womens Health. 2015;15:9. https://doi.org/10.1186/s12905-015-0173-6.

\section{Ready to submit your research? Choose BMC and benefit from:}

- fast, convenient online submission

- thorough peer review by experienced researchers in your field

- rapid publication on acceptance

- support for research data, including large and complex data types

- gold Open Access which fosters wider collaboration and increased citations

- maximum visibility for your research: over $100 \mathrm{M}$ website views per year

At BMC, research is always in progress.

Learn more biomedcentral.com/submissions 